Received: 2017.01 .17

Accepted: 2018.02.07

Published: 2018.02 .24

\title{
Ovarian Reserve Assessment in Celiac Patients of Reproductive Age
}

Authors' Contribution: Study Design A Data Collection B Statistical Analysis C Data Interpretation D Manuscript Preparation E Literature Search F Funds Collection $G$

Corresponding Author: Source of support:
ABCDEFG 1 Erol Cakmak

B 2 Savas Karakus

C 3 Ozlem Demirpence

E 4 Banu Demet Coskun
1 Department of Gastroenterology, Cumhuriyet University Faculty of Medicine, Sivas, Turkey

2 Department of Obstetrics and Gynecology, Cumhuriyet University Faculty of Medicine, Sivas, Turkey

3 Department of Biochemistry, Cumhuriyet University Faculty of Medicine, Sivas, Turkey

4 Department of Gastroenterology, Kayseri Training and Research Hospital, Kayseri, Turkey

Background: This study aimed to investigate ovarian reserve in patients of reproductive age with Celiac disease (CD) using anti-Müllerian hormone (AMH) levels, antral follicle counts (AFCs), and ovarian volume.

Material/Methods: We included into this study $46 \mathrm{CD}$ female patients and 40 healthy female subjects of reproductive age, ages 18-45 years. Venous blood samples were taken from both groups on days 2-4 of the menstrual cycle, and follicle stimulating hormone ( $F S H)$, luteinizing hormone $(\mathrm{LH})$, estradiol $\left(\mathrm{E}_{2}\right)$, prolactin $(\mathrm{PRL})$, and $A M H$ levels were measured. On the same day, AFCs and ovarian volumes were determined. Data on body mass index (BMI), gravidity/parity/abortions/alive counts, disease duration, and Marsh histological classification were recorded.

Results: There were no statistically significant differences between $\mathrm{CD}$ and control groups in terms of mean age, BMI, or median gravidity/parity/abortions/alive counts $(p>0.05)$. Also, there were no statistically significant differences between the 2 groups in terms of mean $\mathrm{FSH}, \mathrm{LH}, \mathrm{E}_{2}, \mathrm{PRL}$ levels, right and left ovarian volumes, and median right and left ovarian AFCs ( $p>0.05)$. However, AMH level was significantly lower in the CD group $(p=0.032)$. No statistically significant correlation was found between AMH levels and age, BMI, FSH, LH, E, PRL levels, right and left ovarian volumes, right and left ovarian AFCs, or Marsh histological classification using the Spearman correlation test $(p>0.05)$. However, an inverse correlation was detected showing that AMH levels decrease with increasing CD duration $(r=-0.054, p=0.001)$.

Conclusions: We found that $\mathrm{AMH}$ level and ovarian reserve was decreased in $\mathrm{CD}$ patients of reproductive age compared to healthy controls, and that $\mathrm{AMH}$ level and ovarian reserve decreased with increasing disease duration in $\mathrm{CD}$ patients.

MeSH Keywords: Anti-Müllerian Hormone • Celiac Disease • Infertility

Full-text PDF: https://www.medscimonit.com/abstract/index/idArt/909033

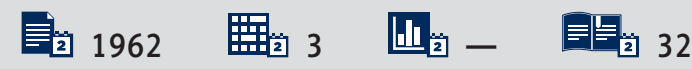




\section{Background}

Celiac disease is an autoimmune disease characterized by the involvement of multiple systems, induced by dietary intake of gluten in genetically-predisposed individuals [1]. In celiac disease, gluten-containing nutrients cause decreased enterocyte height, increased intraepithelial T-lymphocytes, crypt hyperplasia, and villous atrophy in small intestine mucosa, and the absorbing surface of the small intestine dramatically decreases and causes insufficient nutrient absorption [2]. As a result, there occur typical intestinal symptoms such as diarrhea, anorexia, abdominal pain, bloating with vomiting, as well as extraintestinal symptoms such as skin disorders, neuromuscular symptoms, bone disease, and reproductive disorders [3].

The prevalence of Celiac disease is especially greater in women of reproductive age. Celiac disease has adverse effects on both fertility and pregnancy in women of reproductive age. This has been associated with autoimmunity and nutrient malabsorption [4]. Celiac disease causes reproductive disorders, including infertility, delayed menarche, amenorrhea, and early menopause in women of reproductive age. In pregnant women, it causes recurrent abortions, preterm deliveries, intrauterine fetal growth restriction, and low-birth-weight infants $[4,5]$. The cause of these complications in pregnant women is nutritional deficiencies, as well as changed placental functions due to maternal celiac disease auto-antibodies binding to placental transglutaminase and genetic mutations facilitating the formation of microthrombus. Improvement in fertility, miscarriage rates, fetal nutrition, and perinatal course has been observed in these patients upon initiation of a gluten-free diet and recovery of the intestinal mucosa $[5,6]$.

Anti-müllerian hormone (AMH) is a dimeric glycoprotein and member of the transforming growth factor beta family, released by the granulosa cells of pre-antral and early antral follicles. This hormone plays an important role in the regulation of follicle growth [7], and another important function is to slow the reduction of ovarian reserve and to preserve the ovarian reserve. Women of reproductive age with low AMH levels had the lowest ovarian reserve and the highest risk of premature ovarian failure [8]. Therefore, AMH level is the optimal biochemical marker, reflecting the primordial follicle pool and ovarian function. Serum AMH level is recognized as the most useful test for the determination of fertility potential in women of reproductive age. AMH level and primordial follicles decrease with age, and cannot be detected in the menopausal period $[9,10]$.

Our literature search did not find any study exploring the ovarian reserve in celiac patients. The present study aimed to compare ovarian reserves of women with celiac disease of reproductive age and healthy women, using antral follicle counts (AFCs) and ovarian volumes.

\section{Material and Methods}

\section{Patients and data collection}

Our study included 46 female patients with celiac disease and 40 healthy controls with reproductive potential, ages 18-45 years, between January 2016 and October 2017. Study inclusion criteria were having regular menstrual cycles (length of 21-35 days) and both ovaries being present. Exclusion criteria were smoking, premature menopause, chronic renal disease, chronic hepatic disease, malignancy, history of infertility, hormonotherapy affecting ovarian reserve (in the last 6 months), pregnancy, history of surgery, and gynecological problems such as polycystic ovary syndrome.

Subjects included into the study were diagnosed with CD by a combination of medical history, physical examination, serology, and histological analysis of multiple biopsies taken by upper-gastrointestinal endoscopy, and based on ACG guidelines [11]. Duodenal biopsies of the patients with CD were classified based on Marsh-Oberhuber (MO) classification: $0=$ normal mucosa, $1=$ increase in intraepithelial lymphocyte count, $2=$ crypt hyperplasia, $3 a=$ partial villus atrophy, $3 b=$ subtotal villus atrophy, and $3 c=$ total villus atrophy [6].

Venous blood samples were taken from groups on days 2-4 of the menstrual cycle, and follicle stimulating hormone (FSH), luteinizing hormone $(\mathrm{LH})$, estradiol $\left(E_{2}\right)$, prolactin $(P R L)$, and $\mathrm{AMH}$ levels were determined by enzyme-linked immunosorbent assay (ELISA) method. The AMH levels were determined using the AMH ELISA kit (Fine Biological Technology, Wuhan, Hubei, China). FSH, LH, $E_{2}$, and PRL were analyzed by the Chemiluminescent Microparticle Immunoassay (Cobas ${ }^{\circledR}$, Roche, Manheim, Germany).

Ovarian volumes and AFCs were determined by pelvic ultrasonography (USG) performed on the same day. Also, subjects' ovarian volumes were calculated by an ellipsoid equation (len gth $\times$ height $\times$ width $\times 0.526-0.526)$. Participants' height, weight, and body mass index (BMI), disease duration, gravidity, parity, abortions, and number of alive children were determined. This study protocol was approved by Cumhuriyet University Ethics Committee (No: 2015-11/04) and performed according to the Declaration of Helsinki principles.

\section{Statistical analysis}

All statistical analyses were performed using SPSS program 22.0 (Chicago, Illinois, USA). Normality was tested using Kolmogorov-Smirnov test. The $t$ test was performed for variables fitting to normal distribution, and the Mann-Whitney $U$ test was used for variables not fitting to normal distribution. Spearman correlation test was used for the correlation of $\mathrm{AMH}$ 
Table 1. Demographic ve clinical characteristics of the patients and controls.

\begin{tabular}{|c|c|c|c|}
\hline Variables & Control $(n=40)$ & CD $(n=46)$ & $p$-Value \\
\hline Age (years) & $31.5 \pm 9.5$ & $35.2 \pm 7.9$ & $0.059^{*}$ \\
\hline BMI $\left(\mathrm{kg} / \mathrm{m}^{2}\right)$ & $25.1 \pm 3.9$ & $24.6 \pm 3.7$ & $0.625^{*}$ \\
\hline Gravidity (n) & $2(0-7)$ & $2(0-7)$ & $0.084^{\star *}$ \\
\hline Parity (n) & $1 \quad(0-5)$ & $2(0-6)$ & $0.159^{* *}$ \\
\hline Abortions (n) & $0 \quad(0-4)$ & $0 \quad(0-5)$ & $0.238^{\star *}$ \\
\hline Alive (n) & $1(0-5)$ & $2(0-4)$ & $0.140^{* *}$ \\
\hline Age at CD onset (years) & - & $31.5 \pm 8.2$ & - \\
\hline Age at CD diagnosis (years) & - & $32.1 \pm 8.2$ & - \\
\hline Disease duration (years) & - & $4(1-19)$ & - \\
\hline \multicolumn{4}{|c|}{ Histology (Marsh classification) (n, \%) } \\
\hline 1 & - & $5(10.8)$ & - \\
\hline 2 & - & $7(15.2)$ & - \\
\hline 3 & - & $34 \quad(74)$ & - \\
\hline $3 a$ & - & $7 \quad(15.2)$ & - \\
\hline $3 b$ & - & $15 \quad(32.7)$ & - \\
\hline $3 c$ & - & $12(26.1)$ & - \\
\hline
\end{tabular}

* Student's t- test; ${ }^{* \star}$ Mann-Whitney U-test. BMI - body mass index.

levels with other variables. $p$ values $<0.05$ were regarded as statistically significant.

\section{Results}

The mean age of the patients with celiac disease and healthy control subjects were $35.2 \pm 7.9$ and $31.5 \pm 9.5(p=0.059)$, respectively. There was no statistically significant difference between patient and control groups in terms of mean age, BMI, and median number of gravidity/parity/abortions/alive children $(p>0.05)$. The mean disease duration in the CD group was 4 years (range, 1-19 years). Based on CD histological Marsh classification, 5 patients (10.8\%) were grade 1, 7 (15.2\%) were grade $2,7(15.2 \%)$ were grade $3 a, 15(32.7 \%)$ were grade $3 b$, and $12(26.1 \%)$ were grade $3 c$. Demographic and clinical characteristics of the patient and control groups are shown in Table 1.

There was no statistically significant difference between patient and control groups in terms of mean FSH, LH, $\mathrm{E}_{2}, \mathrm{PRL}$ levels, right and left ovarian volumes, and median right and left ovarian AFCs ( $p>0.05)$. However, AMH level was statistically significantly lower in the CD group $(p=0.032)$. Laboratory and ultrasound results of the patient and control groups are shown in Table 2.
There was no statistically significant correlation between $\mathrm{AMH}$ levels and age, BMI, FSH, LH, $E_{2}$, PRL levels, right and left ovarian volumes, and right and left ovarian AFCs using Spearman correlation test ( $p>0.05)$. There was also no statistically significant correlation between AMH levels and Marsh classification in $C D$ patients $(p>0.05)$. However, the decrease in AMH levels with increasing duration of $C D$ disease was found to be highly significant $(r=-0.504$ and $p<0.001)$. The result of Spearman correlation test between disease duration and $\mathrm{AMH}$ levels is shown in Table 3.

\section{Discussion}

This was the first study to assess ovarian reserve in $C D$ and show decrease in ovarian reserve in these patients. Our study assessed ovarian reserve in $C D$ patients and healthy women of reproductive age using $\mathrm{AFCs}, \mathrm{AMH}$, and other serum hormone levels $\left(E_{2}, F S H, L H\right.$, and PRL) and ovarian ultrasound. We compared patients with $C D$ and healthy control women in terms of ovarian reserve. As a result of this study, we detected statistically significant decrease in serum $\mathrm{AMH}$ level and ovarian reserve in patients with $C D$ compared to healthy women. Moreover, we detected an inverse correlation between disease duration and $\mathrm{AMH}$ levels in CD patients. Specifically, we 
Table 2. Laboratory result, AMH levels, ovarian volumes, and antral follicle numbers of the groups.

\begin{tabular}{|c|c|c|c|}
\hline Variables & Control $(n=40)$ & $C D(n=46)$ & $p$-Value \\
\hline $\mathrm{E}_{2}(\mathrm{pg} / \mathrm{mL})$ & $80.7 \pm 39.5$ & $76.1 \pm 23.9$ & 0.972 \\
\hline FSH (IU/L) & $8.7 \pm 6.7$ & $9.6 \pm 8.1$ & 0.681 \\
\hline LH (IU/L) & $10.7 \pm 5.7$ & $9.4 \pm 5.2$ & 0.217 \\
\hline PRL (ng/mL) & $13.1 \pm 5.4$ & $11.5 \pm 5.3$ & 0.061 \\
\hline AMH (pg/mL; median; range) & $1619.5(292-3000)$ & $1184.5 \quad(17-3000)$ & 0.032 \\
\hline Right ovarian volume $\left(\mathrm{cm}^{3}\right)$ & $4.6 \quad(2.1-10.5)$ & $4.7 \quad(0.8-12.3)$ & 0.945 \\
\hline Left ovarian volume $\left(\mathrm{cm}^{3}\right)$ & $4.7 \quad(1.9-9.4)$ & $4.9 \quad(1.2-12.4)$ & 0.665 \\
\hline Right ovarian AFC (n) & $(4-9)$ & $6 \quad(2-15)$ & 0.218 \\
\hline Left ovarian AFC (n) & $(3-10)$ & $(1-13)$ & 0.133 \\
\hline
\end{tabular}

Mann-Whitney U-test. AFC - antral follicle count; AMH - anti-Müllerian hormone; $E_{2}$ - estradiol; FSH - follicle stimulating hormone; $\mathrm{LH}$ - luteinizing hormone; PRL - prolactin.

Tablo 3. Correlation analysis of disease duration and AMH levels.

\begin{tabular}{cccc}
\hline Sperman correlation test & & AMH & Disease duration \\
\hline \multirow{2}{*}{ Serum AMH $(\mathrm{pg} / \mathrm{mL})$} & Correlation coefficient & 1.000 & -0.504 \\
& Significance & - & $<0.001$ \\
\hline
\end{tabular}

$\mathrm{AMH}$ - anti-Müllerian hormone.

detected that $\mathrm{AMH}$ level and ovarian reserve decreases with increasing disease duration in $C D$ patients.

HLA class II DQ2 or DQ8 genes are predisposing variables in celiac disease and account for about $40 \%$ contribute to development of the disease. Furthermore, the development of celiac disease is affected by nutritional factors, including gut microbiota of infants, time and amount of first exposure to gluten, and environmental factors [12]. Being present in and being the alcohol-soluble fraction of gluten taken via nutrients, gliadin has toxic properties. Gliadin triggers inflammatory reaction, especially in epithelial cells of the small intestine lumen, and causes infiltration in lamina propria and atrophy villi [13]. As a result, it causes increase in several celiac-specific auto-antibodies in patients. Combination of celiac-specific serological tests and duodenal biopsy samples is required for diagnosis of celiac disease in adults [14]. As a result of small intestine mucosa inflammation at varying degrees in celiac disease patients, a broad range of classic gastrointestinal symptoms and atypical extraintestinal problems can be present [15]. Being an extraintestinal problem, infertility is highly important, especially in women of reproductive age. The prevalence of celiac disease around the world differs by geographical region; the worldwide prevalence is $1 \%$, and $60-70 \%$ of these patients are women of reproductive age $[16,17]$. Today, the fertility changes caused by celiac disease, together with the possible adverse course in pregnancy and postpartum period, have begun to be understood better. In women of reproductive age with celiac disease, changes such as delayed menarche, amenorrhea, and premature menopause decrease their fertility. This is caused by autoimmunity, and the nutritional deficiency developing in untreated patients [18]. Studies have shown that infertility and unexplained infertility rates are higher in $C D$ patients than in the general population [15-18]. In another study, $4(2.7 \%)$ CD cases were detected in 150 infertile women and none in healthy women [19]. In a similar study in a northern Sardinia population, CD prevalence was $3.03 \%$ in 99 infertile female patients versus $1.06 \%$ in the normal population. Also, in this study, the prevalence of celiac disease in women with unexplained infertility, especially in subgroup analysis, was $8 \%$ [20]. In a study in infertile Arabic women, CD prevalence was $2.65 \%$, and it was $0.5 \%$ in the control group [21]. If female patients of reproductive age have infertility and unexplained infertility, CD investigation is recommended [22]. Kotze detected higher rates of delayed menarche, amenorrhea, and spontaneous abortion in CD patients compared to the control group, regardless of nutritional status [23]. In males and females with celiac disease, recovery of fertility has been observed upon gluten withdrawal and correction of the deficiency of dietary elements with gluten-free diet [24,25]. It is known that immune mechanisms along with nutritional effect play a crucial role in pregnancy-related problems in CD patients [26]. 
A study in the United Kingdom found that in women with celiac disease, the incidences of infertility, stillbirths, and perinatal deaths were increased and these rates improved after initiation of a gluten-free diet [27].

In a woman of reproductive age, fertility is determined by ovarian reserve, which represents both the quality and the quantity of the ovarian follicular pool. It has been reported that ovarian reserve decreases with age and in some autoimmune diseases. The best test used in clinical practice to assess ovarian reserve is the $\mathrm{AMH}$ test [28]. In the literature, no study was found on the effect on $\mathrm{AMH}$ levels in celiac disease patients. Recently, however, several studies have explored the relationship between some of the autoimmune diseases, and fertility and ovarian reserve in women of reproductive age. In their study in women of reproductive age with Behcet's disease, Mont'Alverne et al. detected decreased $\mathrm{AMH}$ level and ovarian reserve, and they reported that Behcet's disease activity contributes to the decrease in ovarian reserve [29]. In a study in female patients with systemic lupus erythematosus, it was reported that $\mathrm{AMH}$ levels are decreased in this disease compared to the healthy population, and active disease has negative effect on ovarian reserve [30]. In another study, a significant decrease was observed in AMH levels in female patients of reproductive age with Crohn's disease compared to the control

\section{References:}

1. Ludvigsson JF, Leffler DA, Bai JC et al: The Oslo definitions for coeliac disease and related terms. Gut, 2013; 62: 43-52

2. Parzanese I, Qehajaj D, Patrinicola F et al: Celiac disease: From pathophysiology to treatment. World J Gastrointest Pathophysiol, 2017; 8: 27-38

3. Tersigni C, Castellani R, de Waure C et al: Celiac disease and reproductive disorders: Meta-analysis of epidemiologic associations and potential pathogenic mechanisms. Hum Reprod Update, 2014; 20: 582-93

4. Eliakim R, Sherer DM: Celiac disease: Fertility and pregnancy. Gynecol Obstet Invest, 2001; 51: 3-7

5. Freeman HJ: Reproductive changes associated with celiac disease. World J Gastroenterol, 2010; 16: 5810-14

6. Oberhuber G, Granditsch G, Vogelsang H: The histopathology of coeliac disease: Time for a standardized report scheme for pathologists. Eur J Gastroenterol Hepatol, 1999; 11: 1185-94

7. Pankhurst MW: A putative role for anti-Müllerian hormone $(A M H)$ in optimising ovarian reserve expenditure. J Endocrinol, 2017; 233: 1-13

8. Seifer DB, Baker VL, Leader B: age-specific serum anti-Müllerian hormone values for 17,120 women presenting to fertility centers within the United States. Fertil Steril, 2011; 95: 747-50

9. Gnoth C, Schuring AN, Friol K et al: Relevance of anti-Müllerian hormone measurement in a routine IVF program. Hum Reprod, 2008; 23: 1359-65

10. Gruijters MJ, Visser JA, Durlinger AL et al: Anti-Müllerian hormone and its role in ovarian function. Mol Cell Endocrinol, 2003; 211: 85-90

11. De Vet A, Laven JS, de Jong FH et al: Anti-Müllerian hormone serum levels: A putative marker for ovarian aging. Fertil Steril, 2002; 77: 357-62

12. Guandalini S, Assiri A: Celiac disease: A review. JAMA Pediatr, 2014; 168: 272-78

13. Green PH, Cellier C: Celiac disease. N Engl J Med, 2007; 357: 1731-43

14. Rubio-Tapia A, Hill ID, Kelly CP et al., American College of Gastroenterology: ACG clinical guidelines: Diagnosis and management of celiac disease. Am J Gastroenterol, 2013; 108: 656-77 group [31]. In FMF patients with M694V mutation, a decrease was detected in AMH levels [32]. These studies show that autoimmune diseases have adverse effects on fertility. Especially, there is an inverse correlation between autoimmune disease activity and ovarian reserve. AMH levels and ovarian reserve decrease with increasing autoimmune disease activity.

The limitation of our study is the low number of patients; however, this is the first study in the literature assessing the ovarian reserve in $C D$ patients of reproductive age. Our findings need to be supported by larger studies.

\section{Conclusions}

We detected decreased $\mathrm{AMH}$ levels and ovarian reserve in $\mathrm{CD}$ patients of reproductive age compared to the healthy control group. Moreover, we detected that AMH levels and ovarian reserve decrease with increasing disease duration, and there is an inverse correlation between them.

\section{Conflicts of interests}

None.

15. Nadhem ON, Azeez G, Smalligan RD et al: Review and practice guidelines for celiac disease in 2014. Postgrad Med, 2015; 127: 259-65

16. Catassi C, Gatti S, Lionetti E: World perspective and celiac disease epidemiology. Dig Dis, 2015; 33: 141-46

17. Shah S, Leffler D: Celiac disease: An underappreciated issue in women's health. Womens Health, 2010; 6: 753-66

18. De Re V, Magris R, Cannizzaro R: New insights into the pathogenesis of celiac disease. Front Med (Lausanne), 2017; 31: 137

19. Collin P, Vilska S, Heinonen PK et al: Infertility and coeliac disease. Gut, 1996; 39: 382-84

20. Meloni GF, Dessole S, Vargiu N et al: The prevalence of coeliac disease in infertility. Hum Reprod, 1999; 14: 2759-61

21. Shamaly H, Mahameed A, Sharony A et al: Infertility and celiac disease: Do we need more than one serological marker? Acta Obstet Gynecol Scand 2004; 83: 1184-88

22. Karaca N, Yılmaz R, Aktun LH et al: Is there any relationship between unrecognized Celiac disease and unexplained infertile couples? Turk J Gastroenterol, 2015; 26: 484-86

23. Kotze LM: Gynecologic and obstetric findings related to nutritional status and adherence to a gluten-free diet in Brazilian patients with celiac disease. J Clin Gastroenterol, 2004; 38: 567-74

24. Sher KS, Jayanthi V, Probert CS et al: Infertility, obstetric and gynaecological problems in coeliac sprue. Dig Dis, 1994; 12: 186-90

25. Lasa JS, Zubiaurre I, Soifer LO: Risk of infertility in patients with celiac disease: A meta-analysis of observational studies. Arq Gastroenterol, 2014; 51: $144-50$

26. Anjum N, Baker PN, Robinson NJ et al: Maternal celiac disease autoantibodies bind directly to syncytiotrophoblast and inhibit placental tissue transglutaminase activity. Reprod Biol Endocrinol, 2009; 7: 16

27. Sher KS, Mayberry JF: Female fertility, obstetric and gynaecological history in coeliac disease. A case control study. Digestion, 1994; 55: 243-46 
28. Broer SL, Broekmans FJ, Laven JS et al: Anti-Müllerian hormone: Ovarian reserve testing and its potential clinical implications. Hum Reprod Update, 2014; 20: 688-701

29. Mont'Alverne AR, Yamakami LY, Gonçalves CR et al: Diminished ovarian reserve in Behçet's disease patients. Clin Rheumatol, 2015; 34: 179-83

30. Martins NFE, Seixas MI, Pereira JP et al: Anti-müllerian hormone and ovarian reserve in systemic lupus erythematosus. Clin Rheumatol, 2017; 36: 2853-54
31. Şenateş E, Çolak Y, Erdem ED et al: Serum anti-Müllerian hormone levels are lower in reproductive-age women with Crohn's disease compared to healthy control women. J Crohns Colitis, 2013; 7: 29-34

32. Şahin A, Karakuş S, Durmaz Y et al: Evaluation of ovarian reserve with antiMüllerian hormone in familial mediterranean fever. Int J Rheumatol, 2015; 2015: 380354 